\title{
High Reynolds number oscillating contact lines
}

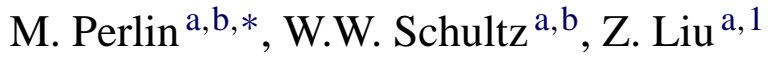 \\ ${ }^{a}$ Department of Naval Architecture and Marine Engineering, University of Michigan, Ann Arbor, MI 48109, USA \\ ${ }^{\mathrm{b}}$ Department of Mechanical Engineering, University of Michigan, Ann Arbor, MI 48109, USA
}

Received 23 July 2003; received in revised form 17 November 2003; accepted 22 December 2003

\begin{abstract}
For the eventual use as regards wave generation and damping, we investigate oscillating contact lines on vertical walls. Stainless steel is used rather than the glass of Ting and Perlin [J. Fluid Mech. 295 (1995) 263] for the vertically sinusoidally oscillating plate over a large range of Reynolds numbers. The non-wetting stainless steel minimizes the static meniscus ignored in our analysis. Other than the change in static contact angle serving as an initial condition, the dynamic features in both cases are similar. In low Reynolds number oscillation, a pinned-edge condition can appropriately describe the contact-line motion. Higher Reynolds number oscillatory contact-line behavior becomes non-linear and very complicated. The resulting periodic, non-sinusoidal oscillation of the contact line exhibits three types of apparent motion: stick (associated with contact angle hysteresis), partial stick where only portions of the oscillation cycle exhibit stick, and total slip. Increasing the Reynolds number reduces hysteresis phenomena, however, it still cannot be ignored. It is seen that the static contact angle is unimportant; it is the dynamic contact angle that is consequential.
\end{abstract}

(c) 2003 Elsevier B.V. All rights reserved.

\section{Introduction}

We study an oscillating contact-line; the tri-junction of a liquid, gas/vapor, and solid that moves to and fro. The interaction/generation of surface waves by surface-piecing bodies has been the subject of many investigations. Often, the effect of surface tension can be ignored safely. When waves are short waves or when the gravitational effect is reduced, capillarity must be included. The presence of capillarity adds an extra term to the dynamic-free surface boundary condition proportional to the free surface curvature and thus increases the order of the dynamic-free surface boundary condition. Hence extra conditions are required at the ends of a finite domain. These conditions determine the position or slope of the free surface at its intersection with the container boundary.

Without surface tension, the idealized-free surface of potential flow is always orthogonal to a vertical wall. However, this condition may be inappropriate if capillarity is present. The extension of an orthogonal condition to waves with capillary forces is a free-end edge condition, implying that the contact angle $\theta$ (see Fig. 1) is fixed at $\pi / 2$, and that the contact line moves freely along the solid boundary. This is not the case.

\footnotetext{
* Corresponding author. Tel.: +1-734-7634754; fax: +1-734-9368820.

E-mail address: perlin@umich.edu (M. Perlin).

${ }^{1}$ Present address: CSO-Aker Engineering Inc., Houston, TX 77079, USA.
} 


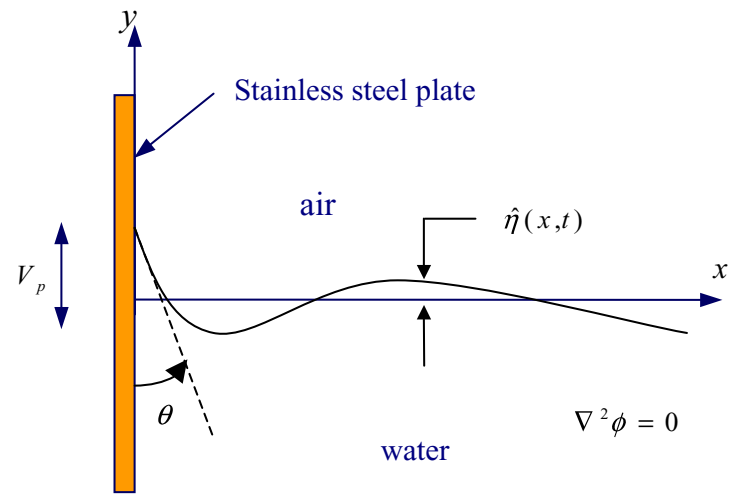

Fig. 1. Sketch of the BVP and the coordinate system.

Several alternative boundary conditions for the edge of a free surface have been proposed. Benjamin and Scott [2] proposed a pinned-end edge condition for a rim-full container. They argued that the same condition could be applied to the interface when the contact angle is within the static range even if the container is not rim-full. Graham-Eagle [3] used the same condition to determine the frequencies of capillary-gravity waves in a rim-full circular cylinder.

Dussan [4] described a model of the relative velocity between a contact line and a solid plate as a function of the contact angle, primarily for a very viscous fluid (high capillary number). In Fig. 2, $\theta_{\mathrm{a}}$ and $\theta_{\mathrm{r}}$ represent the advancing (relative to the plate, the contact line advances in the direction of the gas when the contact angle is greater than $\theta_{\mathrm{a}}$ ) and receding (relative to the plate, the contact line recedes from the gas when the contact angle is less than $\left.\theta_{\mathrm{r}}\right)$ contact angle, respectively. When the contact angle is within $\left(\theta_{\mathrm{r}}, \theta_{\mathrm{a}}\right)$, this model predicts no relative motion between the contact line and the plate; beyond that range, relative motion occurs. Based on the model shown in Fig. 2, Davis [5] discussed five types of contact-line problems and also stated how to pose the contact-line boundary conditions for each, but none of these problems involved oscillating contact lines.

To study capillary-gravity wave damping at a vertical wall (one of our eventual objectives), for low capillary number Hocking [6] proposed the simplest, non-trivial contact-line model for oscillating flows: a linear relationship between the contact-line velocity and the surface slope at the contact point. Two models with and without contact angle hysteresis were presented. Assuming a static or mean-dynamic contact angle of $\pi / 2$, the advancing and receding contact angles are given by $\theta_{\mathrm{a}}=\pi / 2+\alpha_{\mathrm{h}}$ and $\theta_{\mathrm{r}}=\pi / 2-\alpha_{\mathrm{h}}$, where the magnitude of the surface slope at the wall, $|\partial \hat{\eta} / \partial x|_{x=0}$, is assumed small. The simple model to be applied at $x=0$ (shown in Fig. 3 ) is

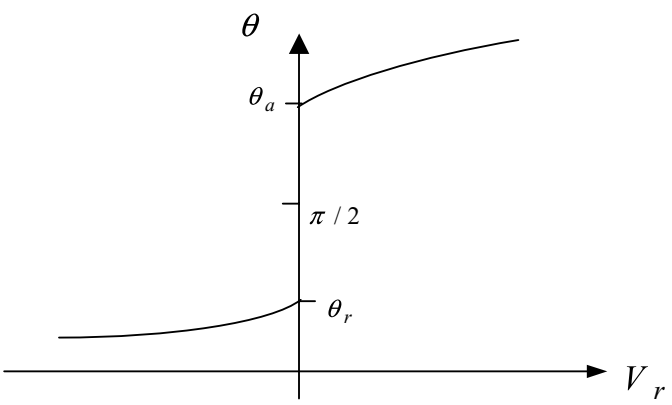

Fig. 2. Contact angle vs. contact-line velocity for unidirectional flow. 


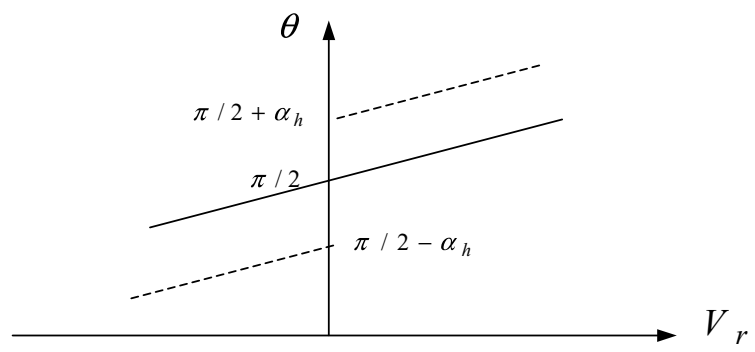

Fig. 3. Contact-line condition with (dash line) and without (solid line) hysteresis (after Hocking [6,7]).

given by

$$
v_{\mathrm{r}}=\frac{\partial \hat{\eta}}{\partial t}-v_{\mathrm{p}}= \begin{cases}\beta\left(\frac{\partial \hat{\eta}}{\partial x}-\alpha_{\mathrm{h}}\right) & \text { if } \frac{\partial \hat{\eta}}{\partial x}>\alpha_{\mathrm{h}}, \\ 0 & \text { if }\left|\frac{\partial \hat{\eta}}{\partial x}\right|<\alpha_{\mathrm{h}}, \\ \beta\left(\frac{\partial \hat{\eta}}{\partial x}+\alpha_{\mathrm{h}}\right) & \text { if } \frac{\partial \hat{\eta}}{\partial x}<-\alpha_{\mathrm{h}},\end{cases}
$$

where $\hat{\eta}$ is the surface elevation, $v_{\mathrm{p}}$ the plate velocity and $\beta^{-1}$ the slope of the two line segments of Fig. 3 . In the remainder of the paper, $v_{\mathrm{r}}, \hat{\eta}, v_{\mathrm{p}}$ are taken as complex variables of a complex exponential representing the temporal oscillation where the real part is physically significant. Henceforth, all variables have been made dimensionless by the length scale $k_{0}^{-1}$ and the time scale $\omega^{-1}$, where $\omega$ is the angular frequency of the wall (wavemaker) and $k_{0}$ the linear wavenumber implicitly given by the dispersion relation

$$
\omega^{2}=g k_{0}+\frac{\sigma}{\rho} k_{0}^{3} .
$$

Here $\sigma$ is the liquid-gas surface tension, $\rho$ the liquid mass density and $g$ the gravitational acceleration.

The special linear case of no contact angle hysteresis [7] is recovered when $\alpha_{\mathrm{h}}=0$. This simplification of Fig. 2 has the same form used by Young and Davis [8] for creeping flows.

These models neglect the static meniscus effect by assuming a $\pi / 2$ static contact angle, significantly simplifying the analyses. However, experimental evidence indicates that the static contact angle is not unique and can be far from $\pi / 2$ depending on the properties of the three phases. In this sense, Miles' [9] inviscid analysis improved Hocking's model by allowing a non-flat static meniscus.

In the model given by (1), the slip coefficient $\beta$ is a real constant. For oscillating flows, Miles [9] modeled a possible phase shift between the surface slope and the relative velocity at the contact-line using a complex-valued coefficient, $c$, when the plate motion is described by $\mathrm{e}^{\mathrm{i} t}$. This represents a different type of hysteresis than that described in Hocking. Miles imposed the following (Navier) slip condition along the plate associated with an edge condition similar to (1)

$$
v-v_{\mathrm{p}}=l_{\mathrm{s}} \frac{\partial v}{\partial x} \quad \text { along } x=0, y<y_{\mathrm{c}}
$$

where $l_{\mathrm{S}}$ is a dimensionless slip length, also a complex function of $\omega ; y_{\mathrm{c}}$ the elevation of the contact-line and $v$ the vertical speed of the fluid. According to Miles [9], the compatibility condition between his complex-valued representation of (1) and (3) is

$$
c=2 \mathrm{i} l_{\mathrm{s}} .
$$




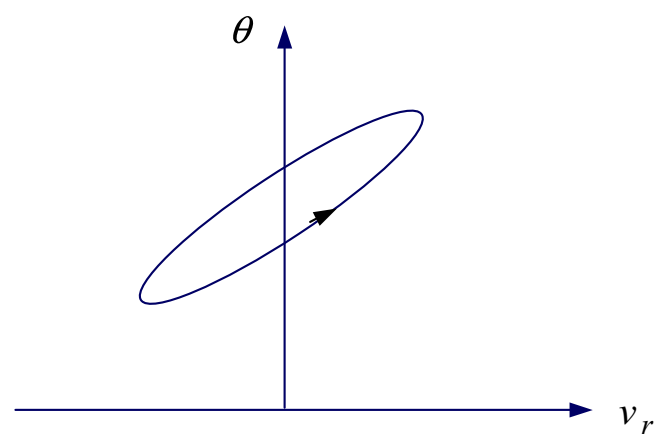

Fig. 4. Miles' model of contact angle vs. relative contact-line velocity for oscillatory flow, $v_{\mathrm{r}}=c \partial \hat{\eta} / \partial x ; c$ is a complex-valued constant. The modulus of $c$ determines the slope of the ellipse, the phase of $c$ determines the area of the ellipse. The ellipse is centered about $\theta_{\mathrm{s}}$ (i.e. the static contact angle) on the vertical axis. Note that $\theta-\pi / 2=\tan ^{-1}(\partial \hat{\eta} / \partial x)$.

To simplify his analysis, Miles assumed that $l_{\mathrm{s}}$ is a complex constant although he acknowledged that this was unrealistic. His assumption of complex constant $c$ leads to a model shown schematically in Fig. 4. The slope of the ellipse major axis is $\beta^{-1}(\beta=|c|)$ while the area is due to the phase. Since the range of $v_{\mathrm{r}}$ is not known a priori the value of $c$ must be iteratively adjusted, unlike the Hocking model.

Our experimental results exhibit a more complicated relationship between the contact angle and contact-line relative velocity. Besides the varying phase shift between the contact angle and the relative velocity of the contact line, $\beta=|c|$ is not constant. Both static and dynamic behavior of the contact line is exhibited during oscillation, requiring the imposed boundary condition to be phase-dependent. We determine $\beta$ by measuring the relationship between the dynamic contact angle and relative contact-line velocity for stainless steel over a large range of $\operatorname{Re}(=$ $\hat{a}^{2} \omega / v=\hat{a} V_{\mathrm{pmax}} / v$ ), where $\hat{a}$ is the dimensional plate stroke amplitude, $\omega$ the radian forcing frequency, $v$ the liquid kinematic viscosity and $V_{\mathrm{p} \text { max }}$ the maximum plate velocity $(\hat{a} \omega)$. The experimental results of a stainless steel plate in contact with a water-air interface show that except for the different static contact angle, the contact-line behavior exhibits similar dynamic features to those of a glass plate in contact with water-air studied earlier by Ting and Perlin [1]. In high Reynolds number oscillation, the contact-line behavior becomes very complicated with three types of motion (slip, partial slip and stick) existing over one period. The dynamic contact angle range exceeds the static range. Contact angle hysteresis decreases with increasing Reynolds number; however, it is never negligible.

Employing a boundary condition similar to (1) with an undetermined slip coefficient model, we solve the 2D inviscid linear boundary value problem in Section 2. Comparing to Miles' solutions [9], two additional terms appear in our surface elevation equation; however, these two terms sum to zero, and therefore we recover Miles' result through a different approach. Retaining all three terms, however, has advantages to be discussed. As expected, comparison to our experimental data indicates that the fixed contact-line boundary condition predicts the contact line behavior well for small Reynolds number oscillation flows only.

In Section 3, we discuss the experimental investigation, including the setup, parameters, and results. Our measurements explore the unique features of the contact-line behavior during high Reynolds number oscillation. One interesting observation is that the static contact angle measured after each experiment is close to the averaged dynamic contact angle over one period.

Finally, we discuss the viscous effect, the non-linear effect, and the material effect on contact-line behavior. According to our experimental parameters, the viscous force can be neglected, i.e. the inviscid assumption is valid. Generally, the nonlinear effects associated with large amplitude or high frequency cause slip, dramatically changing the contact-line behavior. In a reduced gravity environment the nonlinear effect is mitigated, while in a gravity-dominated environment, the non-linear effect is amplified. In spite of the different static contact angles on stainless steel and on glass, similar dynamic features of the contact line are exhibited. This confirms our conjecture 
that the material characteristics affecting the static contact angle can be separated from the dynamic surface boundary condition to simplify analysis greatly.

\section{An alternate derivation of Miles' [9] equations}

In this section, we derive the inviscid 2D boundary value problem for a vertically oscillating upright flat plate with both pinned-contact-line and slip-contact-line conditions. Employing the Fourier-cosine transformation, Hocking [7] solved the inviscid 2D boundary value problem with a linear relationship between the surface slope at the contact point and the relative contact-line velocity (i.e. no hysteresis). From his surface elevation equation, the surface slope at the contact point always equals zero, giving a $\pi / 2$ dynamic contact angle, in contrast to the more complex physical phenomenon typically observed. We employ the same method as Henderson and Miles [10] for studying the surface-wave damping in a circular cylinder with a fixed contact line, providing a dynamic contact angle dependent on the slip coefficient $c$ and the capillary number $\kappa$ that is identical to Miles [9].

We consider an upright plate oscillating vertically and sinusoidally in deep water,

$$
v_{\mathrm{p}}=\mathrm{i} a \mathrm{e}^{\mathrm{i} t} \quad \text { at } x=0,
$$

where $a$ is the prescribed dimensionless oscillation amplitude. A definition sketch with dimensional variables is shown in Fig. 1.

Pursuing temporally periodic solutions, we write the velocity potential as $\phi(x, y) \mathrm{e}^{\mathrm{i} t}$ and the surface elevation as $\eta(x) \mathrm{e}^{\mathrm{i} t}$. The dimensionless linearized 2D inviscid boundary value problem for small amplitude is

$$
\text { governing equation : } \quad \nabla^{2} \phi=0 \text {, }
$$

wall condition : $\quad \frac{\partial \phi}{\partial x}=0 \quad$ on $x=0, y \leq 0$,

kinematic surface condition : $\quad \frac{\partial \phi}{\partial y}=\mathrm{i} \eta \quad$ on $x>0, y=0$,

dynamic surface condition : $\quad \mathrm{i} \phi=\frac{\kappa^{2}}{1+\kappa^{2}} \frac{\partial^{2} \eta}{\partial x^{2}}-\frac{1}{1+\kappa^{2}} \eta \quad$ on $x \geq 0, y=0$,

contact-line condition : $\quad \eta=-\mathrm{i} c \frac{\partial \eta}{\partial x}+a \quad$ on $x=0, y=0$,

with a deep water condition requiring wave motion to decay as $y \rightarrow-\infty$, and a radiation condition requiring only right-going waves as $x \rightarrow \infty$. Here we have imposed a contact condition at the intersection of the free surface and the solid surface with an undetermined complex-valued slip coefficient $c$ that becomes a no-slip boundary condition in the limit $c \rightarrow 0$. The parameter, $\kappa$, is an inverse Bond number defined as $\kappa=(\sigma / \rho g)^{1 / 2} k_{0}$, where $k_{0}$ is a dimensional wavenumber and the term multiplying it is a capillary length. This parameter represents the ratio of capillary to gravitational forces. The details of the solution are similar to those in Henderson and Miles [10] and are given in [11]. The velocity potential and surface elevation are:

$$
\begin{aligned}
& \phi=\frac{2 \mathrm{i}(a-\mathrm{i} c \vartheta) \kappa}{\pi I} \int_{0}^{\infty} \frac{\cos k x \mathrm{e}^{k y} \mathrm{~d} k}{(1-k)\left(\kappa^{2} k^{2}+\kappa^{2} k+\kappa^{2}+1\right)}, \\
& \eta=\frac{2(a-\mathrm{i} c \vartheta) \kappa}{\pi I}\left\{\int_{0}^{\infty} \frac{k \cos k x \mathrm{~d} k}{\left(\kappa^{2} k^{2}+\kappa^{2} k+\kappa^{2}+1\right)(1-k)}+\int_{0}^{\infty} \frac{\cos k x \mathrm{~d} k}{1+\kappa^{2} k^{2}}\right\}-\frac{a-\mathrm{i} c \vartheta}{I} \mathrm{e}^{-x / \kappa},
\end{aligned}
$$

where $k$ is the dimensionless wavenumber and $I$ is given by

$$
I=\frac{2 \kappa}{\pi} \int_{0}^{\infty} \frac{k \mathrm{~d} k}{(1-k)\left(\kappa^{2} k^{2}+\kappa^{2} k+\kappa^{2}+1\right)} .
$$


The last two terms in (8) sum identically to zero. Then (7) and (8) are Miles' [9] equations; however, in its present form, (8) has two advantages: the two integrals within the braces are simpler to compute numerically and the contact-line condition is explicitly met by the final term. Taking the derivative of (8) with respect to $x$ yields the free surface slope at the wall

$$
\vartheta=\frac{\partial \eta}{\partial x}=\frac{a}{\kappa I+\mathrm{i} c} \quad \text { on } x=0 .
$$

In most analyses, a $\pi / 2$ static contact angle is assumed implying a flat static surface. Miles [9] provided the inviscid solution with a static meniscus correction valid for $|\eta| \ll 1$. For $\kappa \ll 1$, his solution of the dynamic contact angle simplifies to

$$
\vartheta=\frac{\left(\hat{a} / l_{\mathrm{c}}\right) \mathrm{e}^{\mathrm{i} \omega t}}{I+\mathrm{i} \gamma-y_{\mathrm{c}} M},
$$

where $\hat{a}$ is the dimensional wave amplitude, $I=-1+((\kappa / \pi)(2 \ln \kappa+1))+2 \pi \mathrm{i}+\mathrm{O}\left(\kappa^{2}\right), M=0.222+\mathrm{O}(\kappa), \gamma=$ $c / \kappa$ and $l_{\mathrm{c}}=\sqrt{\sigma / \rho g}$.

According to the slip condition (1), the slip coefficient $c$, is defined as

$$
c=\frac{\partial \hat{\eta} / \partial t-v_{\mathrm{p}}}{\partial \hat{\eta} / \partial x}=\frac{v_{\mathrm{r}}}{\partial \hat{\eta} / \partial x} .
$$

Hence, $\beta$, the modulus of $c$, is a model parameter specifying the relationship between the contact-line relative velocity and the surface slope at the contact point. Hocking used a real constant $\beta$ in his linear slip condition, which was determined for slow unidirectional flow but applied it to oscillatory flow. Although there are no universal contact-line models, experiments and analysis for capillary spreading demonstrate more complicated relationships between the contact angle and contact-line velocity than shown in Fig. 1. Miles [9] argued that the Hocking real $\beta$ assumption is inappropriate for oscillatory flows and suggested the complex constant, $c$, to allow a phase shift. However, our experimental data show that the contact-line model for the oscillatory flow is also much more complicated than Miles' contact-line model.

\section{Experimental investigation}

\subsection{Experimental setup and parameters}

We measure the dynamic contact angle and the free surface position along a vertically oscillated, upright plate partially immersed in a rectangular tank. The laboratory experiment utilizes a $5 \mathrm{~W}$ Argon-Ion laser; attendant optics (a spherical lens, a cylindrical lens, and several dielectric mirrors); a rectangular glass tank; a stainless steel plate; an electrodynamic shaker with feedback; a water treatment system; and a high-speed intensified video imager. The tank is $610 \mathrm{~mm} \times 305 \mathrm{~mm} \times 495 \mathrm{~mm}$ (length $\times$ width $\times$ height). The plate size is $289 \mathrm{~mm} \times 187 \mathrm{~mm} \times 2 \mathrm{~mm}$ (length $\times$ width $\times$ thickness), immersed approximately $160 \mathrm{~mm}$ into the water. To help avoid surface contamination, the tank and the plate are scrubbed with ethyl alcohol before filling the tank with treated water (i.e. $5 \mu \mathrm{m}$ particulate pre-filtration, carbon adsorption, de-ionization, and $0.2 \mu \mathrm{m}$ particulate filtration). The shaker control includes a Mac PPC computer enhanced with National Instruments' LabVIEW software and data acquisition and output boards.

The setup is shown in Fig. 5. To avoid a reflected image from the water surface, the imager view angle is elevated about $10^{\circ}$ above the surface. We record images of a precise target to determine the image distortion and the system resolution. Results show that the distortion is negligible and the present resolution is $55.4 \mu \mathrm{m} / \mathrm{pixel}$. Fig. 6 is a typical image of the dynamic contact angle and the results of the plate and surface position detection. 


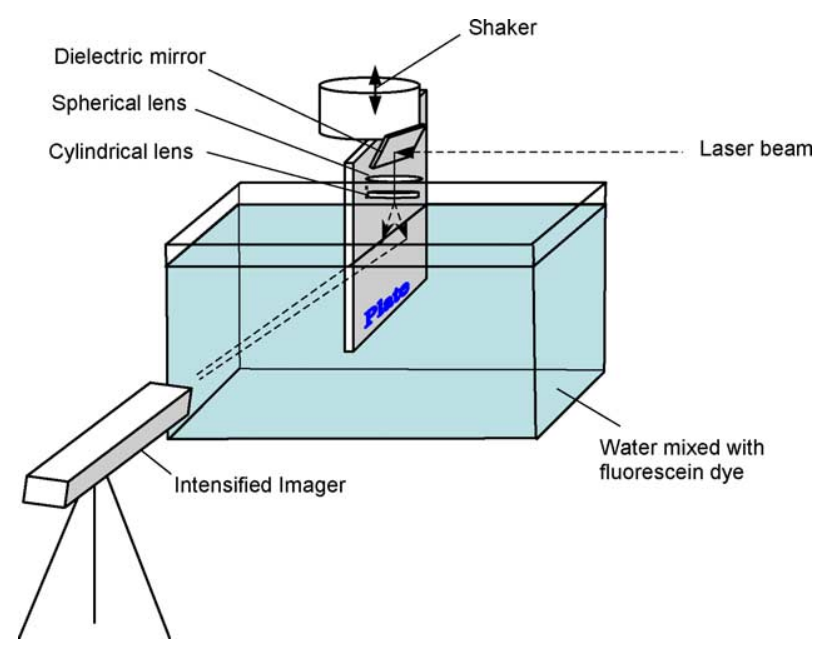

Fig. 5. Experimental setup (not to scale).

The position of the stainless steel plate, the contact line, and the contact angle are required for subsequent analysis, and are determined from the recorded images as follows. A reference point of silicone mixed with rhodamine dye that fluoresces red under the laser light was affixed to the plate immediately above the highest point achieved by the fluid during any of the oscillatory motions of the plate. This referenced the plate position as a function of time. The fluid was dye laden with fluorescein so that it fluoresced blue-green when illuminated by the Argon-Ion laser, thus facilitating location of the meniscus and hence the position of the contact line as a function of time. A protractor was used to measure the apparent contact angle from the laser-illuminated free surface in the recorded images.

In our experiments, six frequencies, 2, 4, 6, 8, 12 and $16 \mathrm{~Hz}$, are selected with seven stroke amplitudes, 0.5, 1, 2, 3, 4, 5 and $6 \mathrm{~mm}$ to cover a large range of Reynolds numbers (see Table 1).

Abundant experimental evidence indicates that a unique static contact angle does not exist in general. It varies within a certain range, depending on the material characteristics and the Young-Laplace angle. When the stainless steel plate is placed in quiescent water, its static contact angle is approximately $90^{\circ}$. Slowly lifting or immersing the plate slightly causes a decrease or increase in the contact angle, respectively. We measure the static hysteresis range

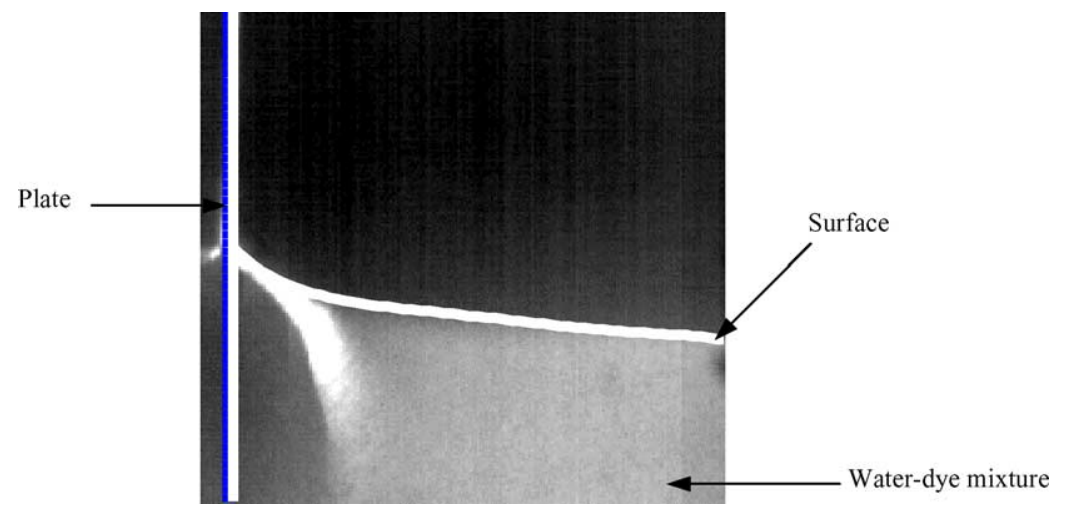

Fig. 6. A snapshot of the surface profile during a $4 \mathrm{~Hz}-1 \mathrm{~mm}$ oscillation. The white solid line and curve indicate the image processing locations of the plate and surface elevation. 
for the stainless steel plate by moving the plate up and down very slowly (i.e. quasi-statically). We also measure the static contact angle at the conclusion of each experiment. Measurements show that the static contact angle of each experiment at its conclusion is approximately $30-40^{\circ}$ (see Table 2), much smaller than the centered-angle $\left(62^{\circ}\right)$ of the static hysteresis range, but close to the average value of the dynamic contact angle over one period.

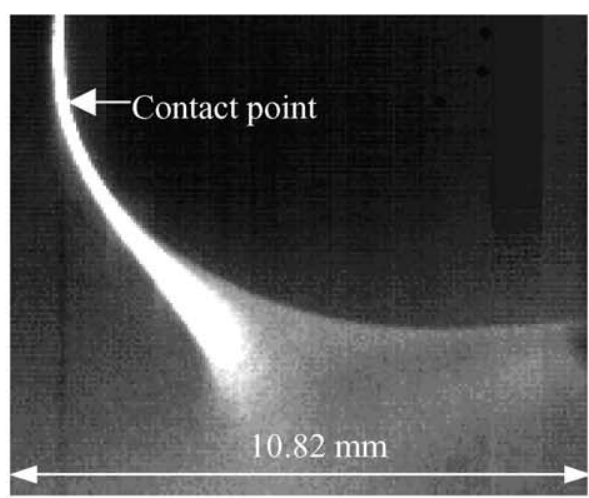

$\omega t=0.0$

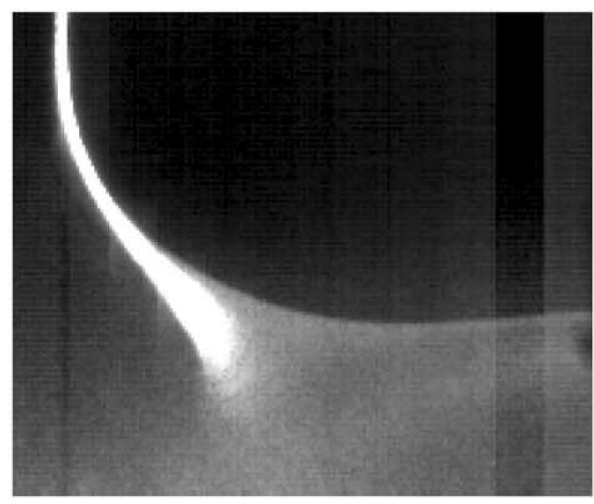

$\omega t=0.4$

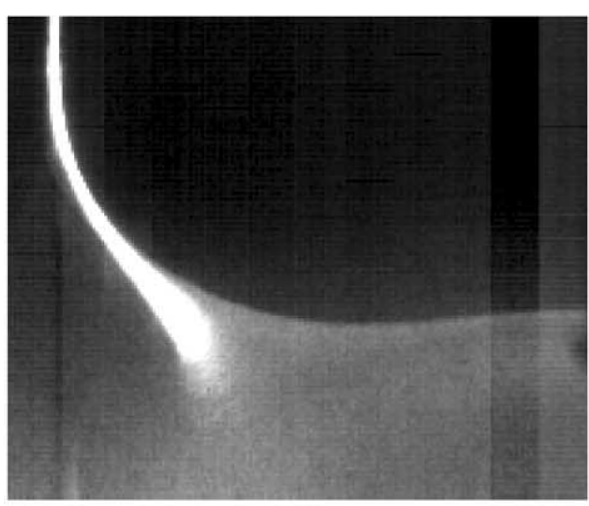

(a)

$$
\omega t=0.8
$$

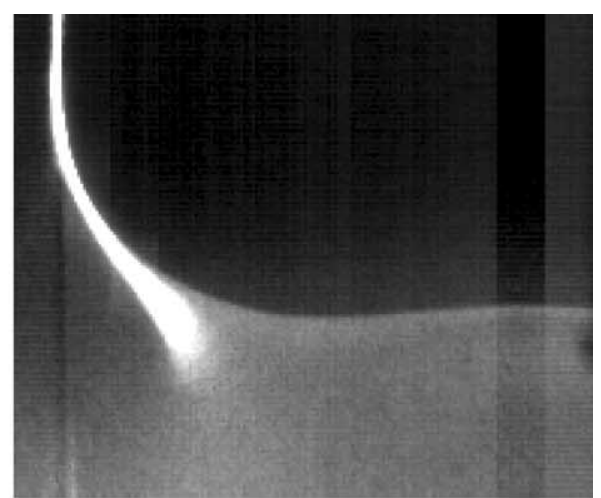

$\omega t=1.2$

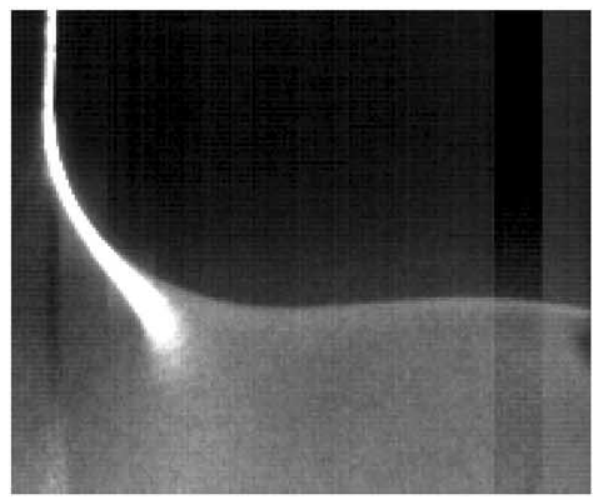

$\omega t=1.6$

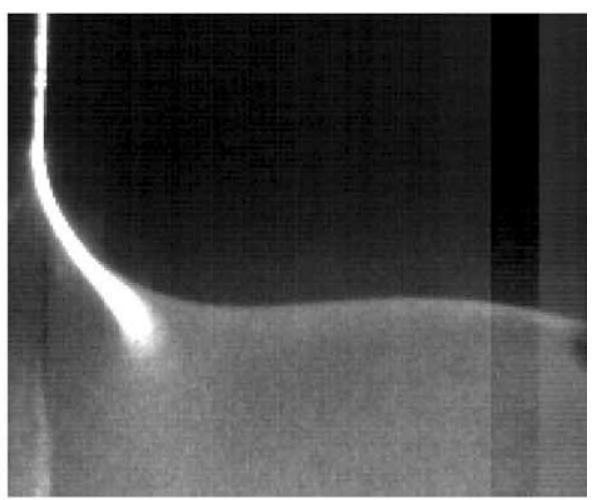

$\omega t=2.0$

Fig. 7. Surface variation during one period of oscillation. The plate motion is $\hat{y}=\hat{a} \cos \omega \hat{t}$, where $\hat{a}=2 \mathrm{~mm}$ and $\omega=32 \pi \mathrm{rad} / \mathrm{s}$. 


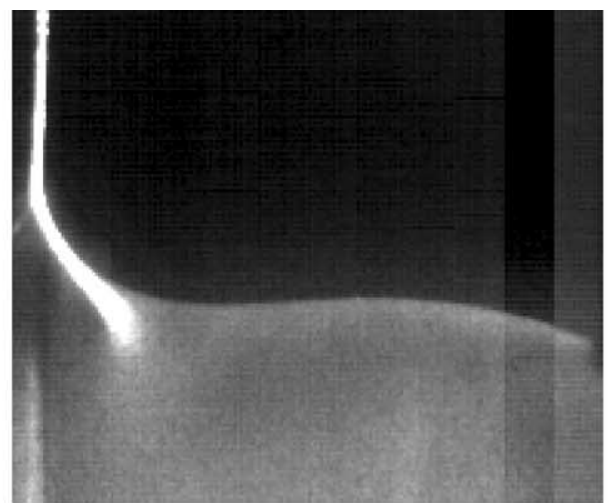

$\omega t=2.4$

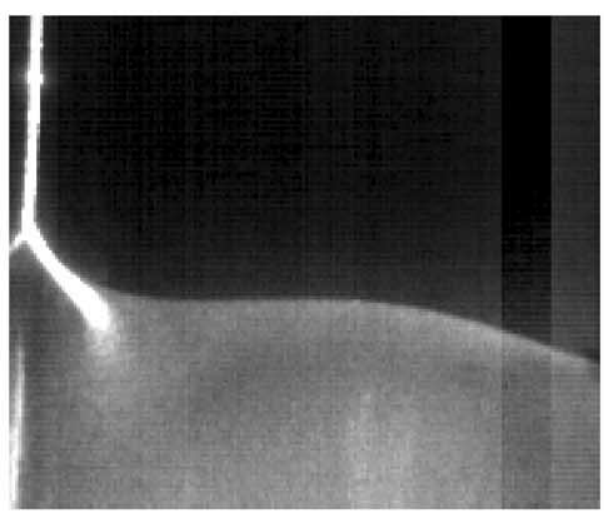

$\omega t=2.8$

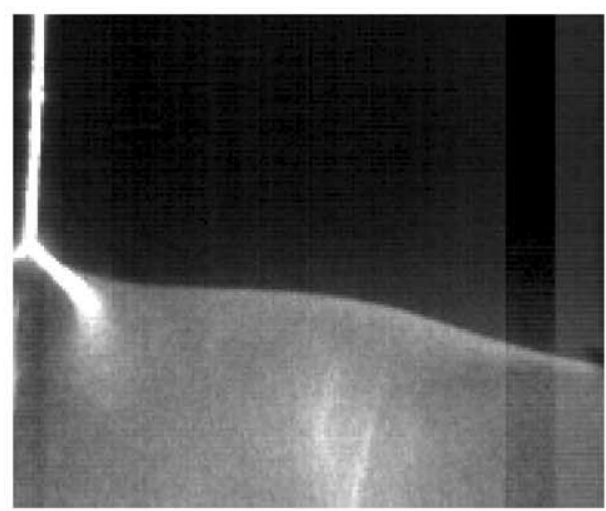

(b)

$$
\omega t=3.2
$$

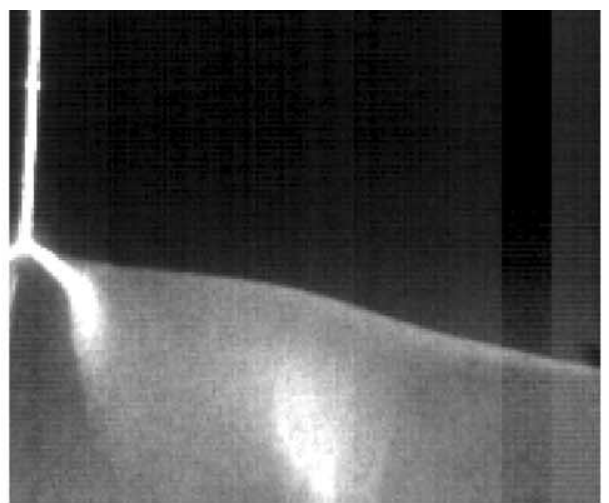

$\omega t=3.6$

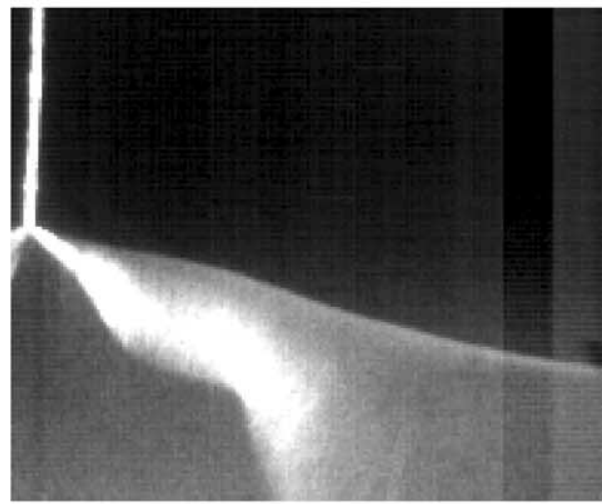

$\omega t=4.0$

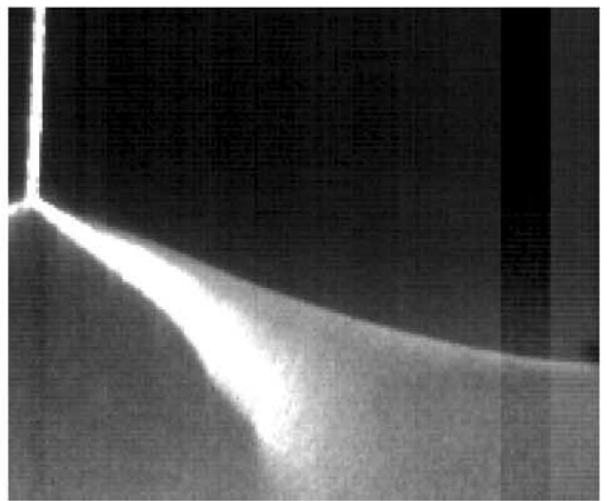

$\omega t=4.4$

Fig. 7. (Continued) 


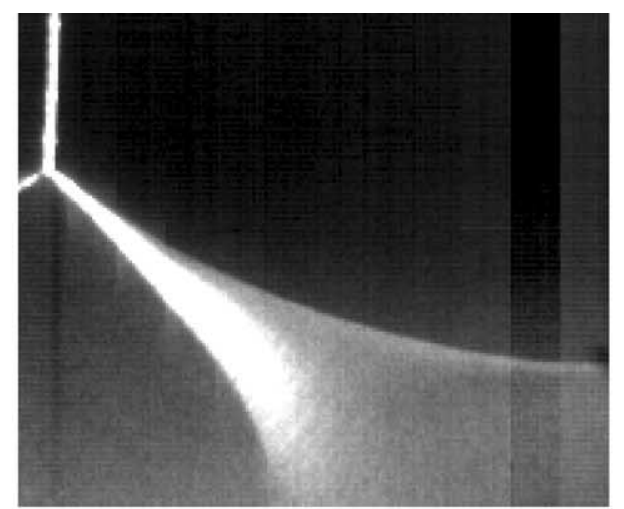

$\omega t=4.8$

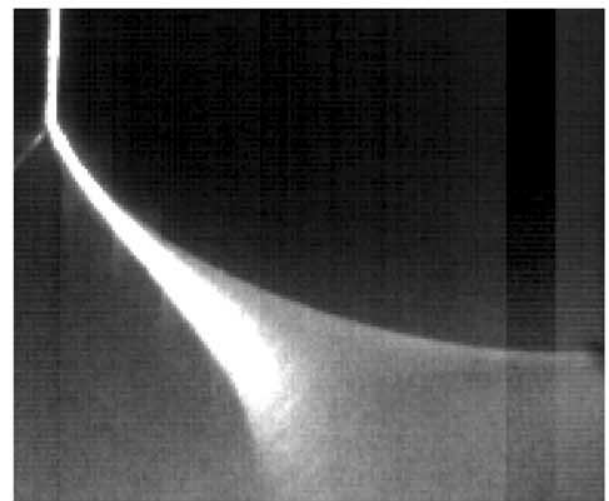

$\omega t=5.2$

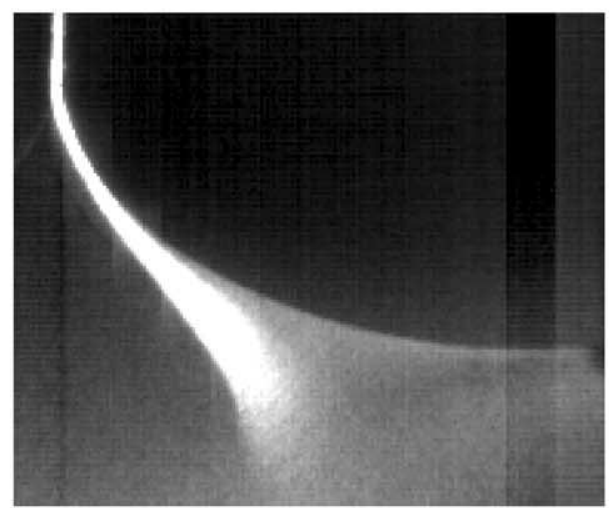

(c)

$\omega t=5.6$

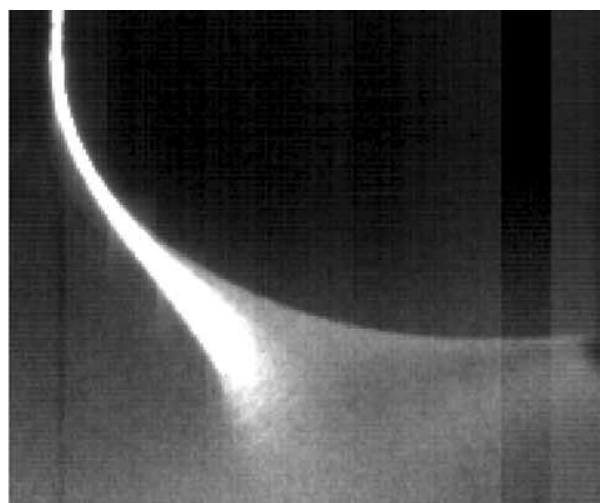

$\omega t=6.0$

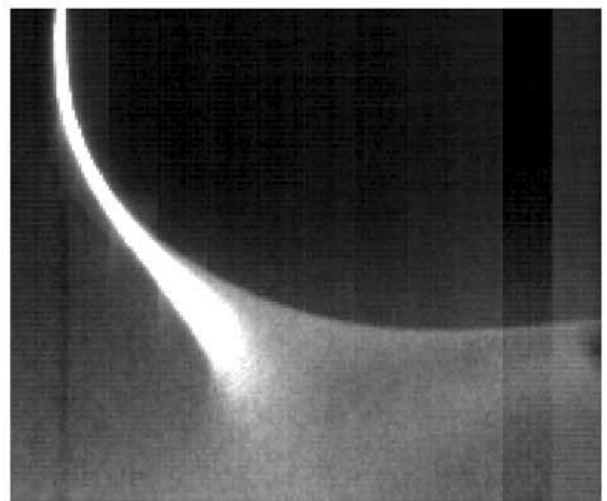

$\omega t=6.4$

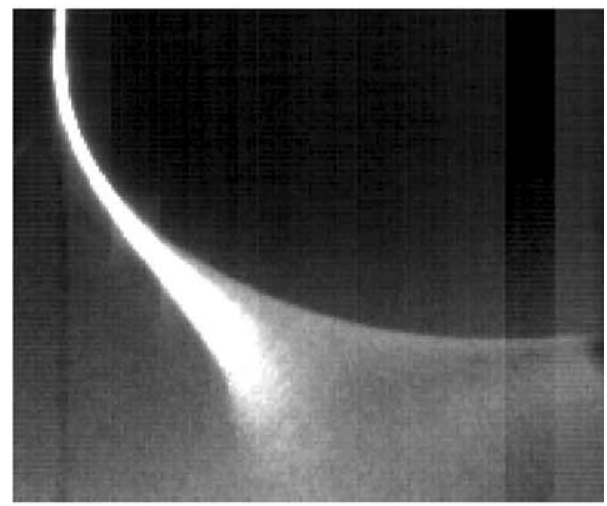

$\omega t=6.8$

Fig. 7. (Continued). 
Table 1

Reynolds number for each experiment, defined as $\left(\hat{a} V_{\mathrm{p} \max }\right) / v$

\begin{tabular}{|c|c|c|c|c|c|c|c|}
\hline \multirow[t]{2}{*}{ Frequency (Hz) } & \multicolumn{7}{|c|}{ Approximate stroke amplitude (mm) } \\
\hline & 0.5 & 1 & 2 & 3 & 4 & 5 & 6 \\
\hline 2 & 2.57 & 10.27 & 41.15 & 95.86 & 175.6 & 276.5 & 385.1 \\
\hline 4 & 5.60 & 21.31 & 88.10 & 193.7 & 351.6 & 517.1 & 780.0 \\
\hline 6 & 8.47 & 31.68 & 122.9 & 287.5 & 500.3 & 812.6 & 1198.0 \\
\hline 8 & 10.81 & 42.62 & 177.1 & 415.8 & 727.3 & 1107.0 & 1630.0 \\
\hline 12 & 16.22 & 62.39 & 257.6 & 586.0 & 1081.0 & - & - \\
\hline 16 & 20.99 & 72.11 & 330.3 & 543.0 & - & - & - \\
\hline
\end{tabular}

$V_{\mathrm{p} \max }$ is the maximum plate velocity, $\hat{a}$ the dimensional stroke amplitude and $v$ the kinematic viscosity of the water-fluorescein mixture.
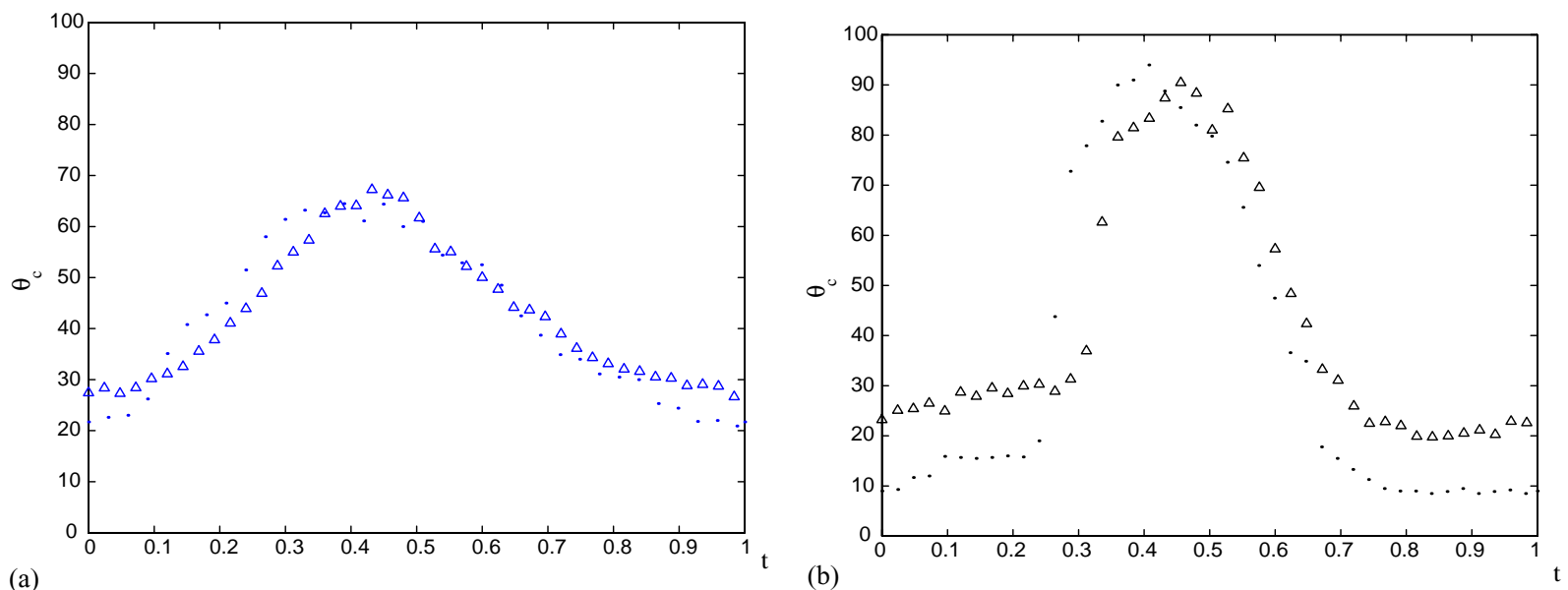

(a)

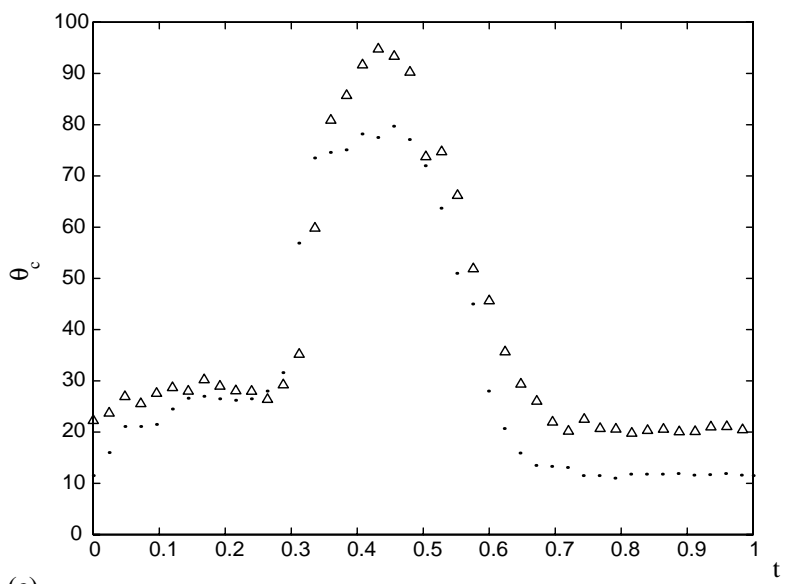

(c)

Fig. 8. Dynamic contact angle $\theta_{\mathrm{c}}$ variation for a $6 \mathrm{~Hz}$ oscillation: $4 \mathrm{~mm}$ and (c) $5 \mathrm{~mm}$. 
Table 2

Static contact angle $\left(^{\circ}\right)$ at the conclusion of each experiment

\begin{tabular}{|c|c|c|c|c|c|c|c|}
\hline \multirow[t]{2}{*}{ Frequency $(\mathrm{Hz})$} & \multicolumn{7}{|c|}{ Approximate stroke amplitude (mm) } \\
\hline & 0.5 & 1 & 2 & 3 & 4 & 5 & 6 \\
\hline 2 & - & 40.36 & 39.34 & 38.71 & 36.11 & 35.38 & 35.20 \\
\hline 4 & 37.64 & 43.37 & 37.01 & 36.04 & 33.73 & 37.47 & 38.84 \\
\hline 6 & 38.34 & 38.80 & 32.28 & 32.71 & 32.96 & 32.79 & 31.29 \\
\hline 8 & 39.01 & 33.85 & 33.82 & 31.86 & 32.54 & 36.28 & 33.16 \\
\hline 12 & 31.52 & 32.56 & 35.32 & 31.98 & 34.57 & - & - \\
\hline 16 & 37.26 & 39.94 & 32.15 & 32.81 & - & - & - \\
\hline
\end{tabular}

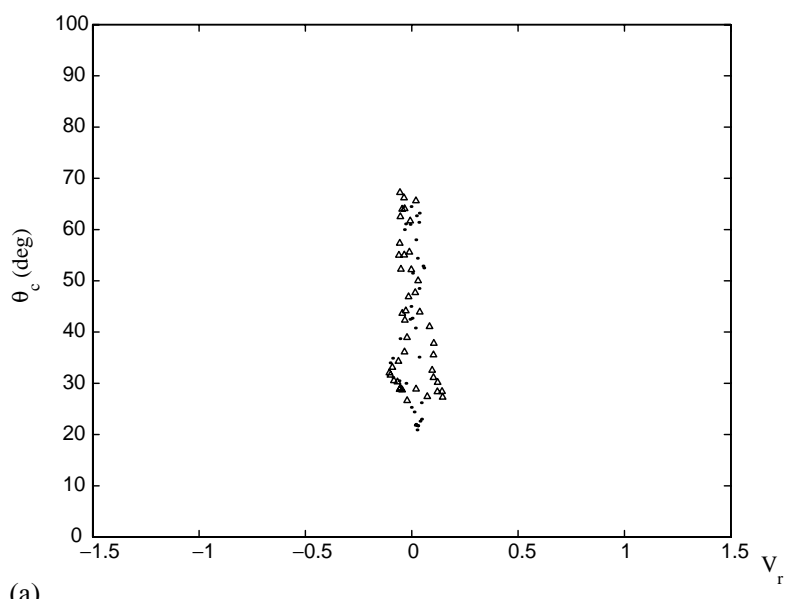

(a)

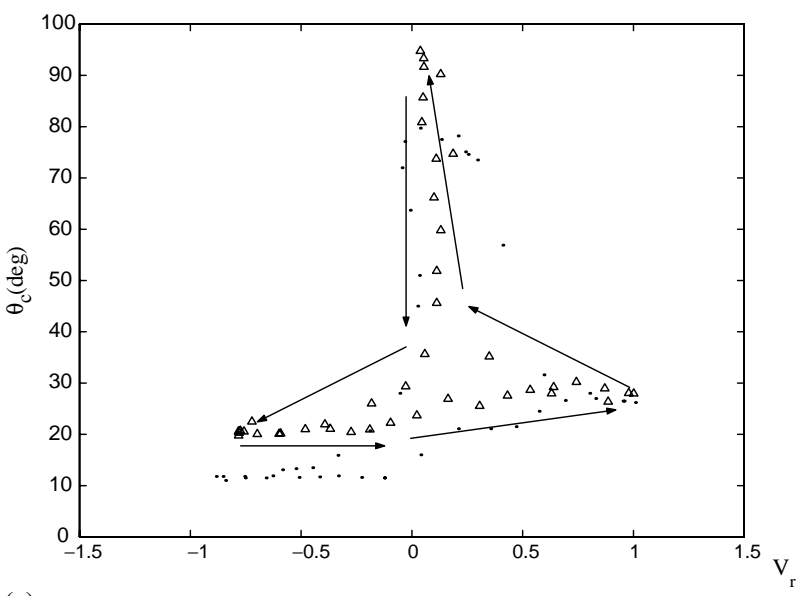

(c)

Fig. 9. Dynamic contact angle $\theta_{\mathrm{c}}$ vs. contact-line relative velocity $V_{\mathrm{r}}$ for a $6 \mathrm{~Hz}$ oscillation: amplitudes: (a) $1 \mathrm{~mm}$, (b) $4 \mathrm{~mm}$ and (c) $5 \mathrm{~mm}$. Arrows in (c) indicate increasing time.

(b)

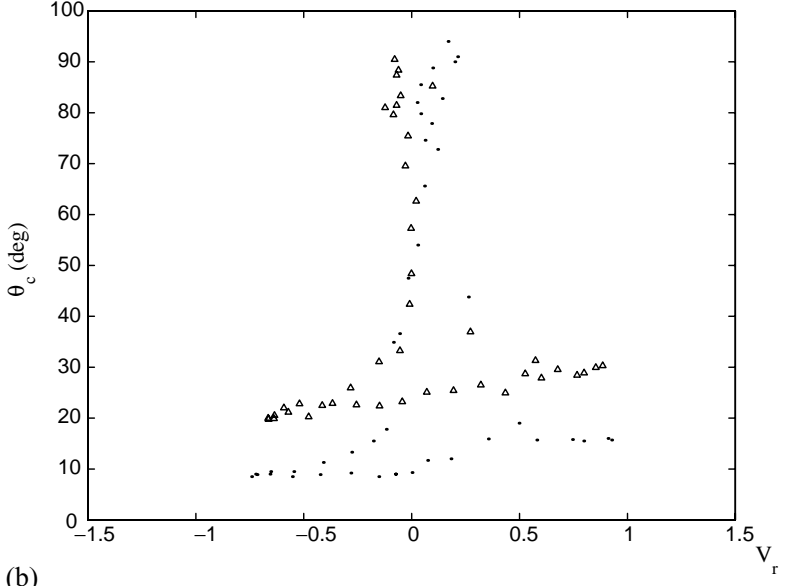




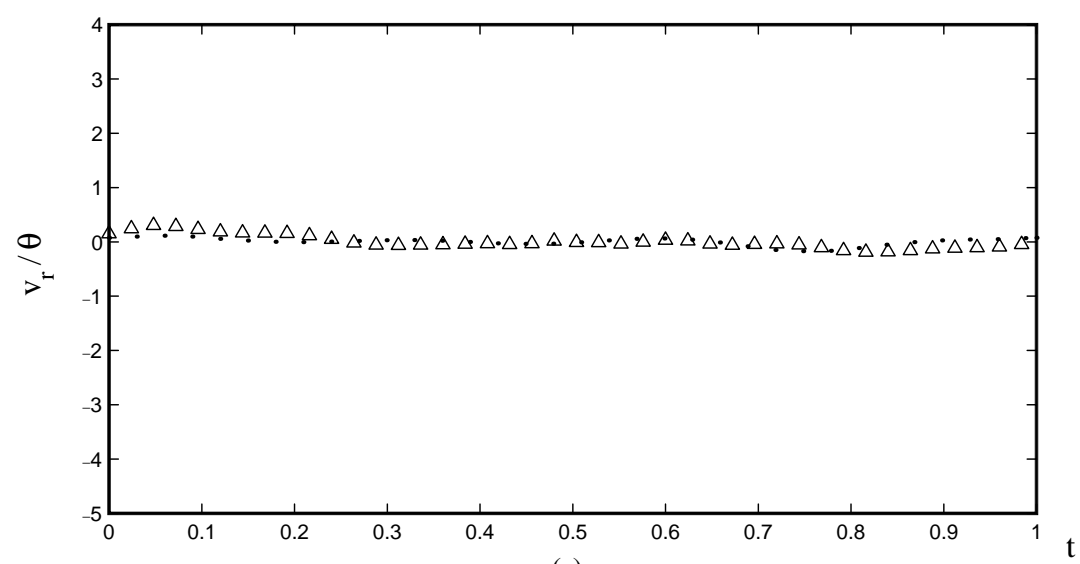

(a)

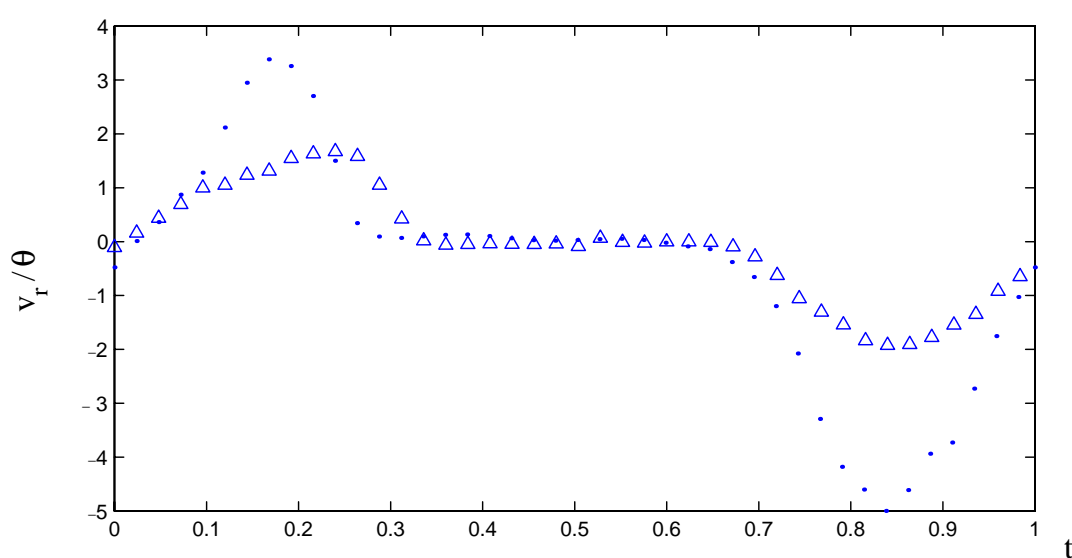

(b)

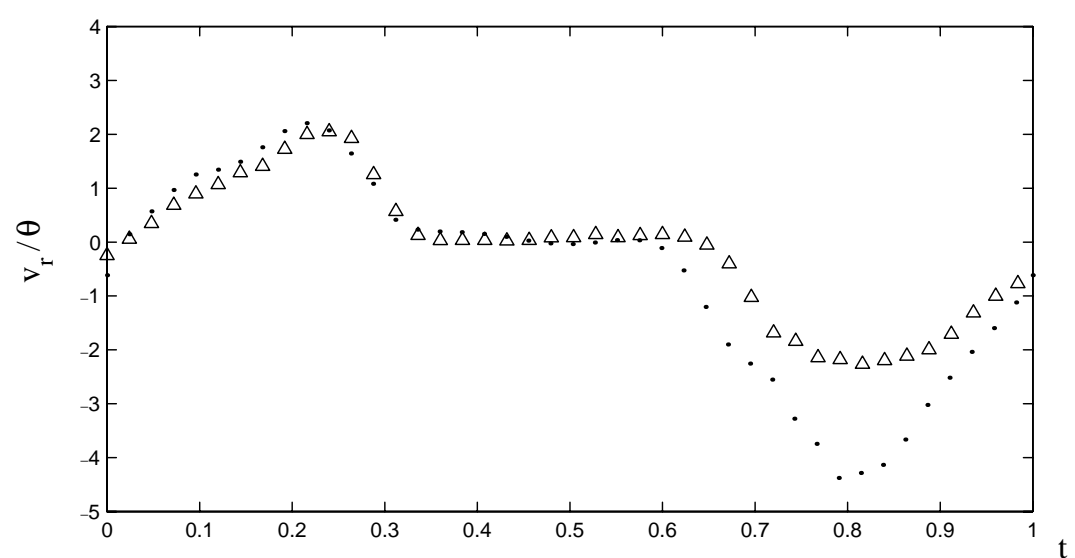

(c)

Fig. 10. $V_{\mathrm{r}} / \theta$ variation over a period of a $6 \mathrm{~Hz}$ oscillation: and (c) $5 \mathrm{~mm}$. 


\subsection{Experimental results}

The primary goals of these experiments are to determine the slip coefficient and to compare the contact-line dynamic behavior of water and a stainless steel plate to water and a glass plate. The dynamic features of the contact-line and contact angle behavior are studied over a large range of Reynolds number. In general, three modes of contact-line motion are observed. First, stick motion, the contact line is fixed relative to the plate. Stick occurs for low amplitude or low frequency oscillations. In this mode, the dynamic contact angle changes within the static range (i.e. $V_{\mathrm{r}}=0$ ) and there is no relative displacement between the plate and the contact line. Second, partial stick-slip motion, relative displacement appears between the liquid and the solid surface accompanied with rapidly changing dynamic contact angle. Third, slip motion, the contact line moves freely along the plate with a barely varying contact angle. Usually stick motion is associated with small Reynolds number oscillation, while in high Reynolds number oscillation all three motions and hysteresis are observed. Images of one period of $16 \mathrm{~Hz}-2 \mathrm{~mm}$ oscillation are shown in Fig. 7 with all three motions observed: slip occurs near the plate maximum position; stick occurs near the plate minimum position; between is the transition (partial slip) motion. Note that the associated surface wave is generated essentially by contact-line motion.

Fig. 8(a)-(c) shows three typical measurements of the dynamic contact angle variation over one period of oscillation for both the stainless steel plate and glass plate [1]. Our measurements show that the receding contact angle changes gradually while the advancing contact angle changes more rapidly as also shown by Ting and Perlin. In the slip mode, the contact angle barely changes. The corresponding dynamic contact angle versus contact-line relative velocity $V_{\mathrm{r}}$ is presented in Fig. 9(a)-(c), where $V_{\mathrm{r}}$ is scaled by the maximum plate velocity. The Reynolds numbers of the stainless steel plate experiments are 31.68, 500.3 and 812.7, respectively; the Reynolds numbers of the glass plate experiments are 40.1, 648.3 and 1023.1, respectively. The contact line exhibits distinct features in these three cases; however, our stainless steel plate measurements and those of Ting and Perlin show remarkable characteristic agreement. In Fig. 9(a), the small amplitude oscillation, no obvious dynamic behavior of the contact line is observed. The contact angle varies within the static range, and the relative velocity between the contact line and the plate is almost zero. Increasing the stroke amplitude causes all three modes of motion over one period, see Fig. 9(b) and (c). When slip occurs, the dynamic contact angle range exceeds the static range. The measurements also show that the contact-line motion is non-sinusoidal, but still periodic in high-Reynolds number oscillations. The relationship between the contact angle and relative contact-line velocity is very different from the complex constant model suggested by Miles [9], see Fig. 4.

The slip coefficient $\beta$, the relationship between the contact angle and the contact-line slope, can be determined experimentally. The $\beta$ corresponding to the data in Fig. 8 is presented in Fig. 10. Results show that in low Reynolds number experiments $\beta$ is zero when only stick motion exists; in high Reynolds number experiments $\beta$ changes form with the different types of motion. It is a function of time, stroke amplitude and frequency during the slip interval and becomes zero during stick motion.

Experimental evidence shows that for high amplitude or high frequency oscillations, both the static and dynamic behavior should be included in the edge condition, requiring the imposed boundary condition change form during the motion. Neglecting the frequency effect, Ting and Perlin [1] proposed a piecewise slip coefficient model that was difficult to implement. An alternative method, suggested by Davis [12], is to neglect the static hysteresis when its range is negligible, which is not appropriate here. According to our experimental data, neither the range of the hysteresis nor its time duration is negligible for high $\operatorname{Re}\left(=\hat{a}^{2} \omega / v\right)$ oscillations.

\section{Discussion and concluding remarks}

To better physically approximate the linear problem, materials are chosen to minimize the meniscus. By comparing experimental results for a stainless steel plate with Ting and Perlin's measurements [1] with a glass plate, we find that in spite of the different static contact angles, contact lines in both experiments exhibit similar dynamic features. 
This confirms our conjecture that material characteristics associated with the static contact angle do not significantly affect the dynamic solution. Hence, the oscillating lubricating liquid layer isolates the flow from the solid material surface.

Our experiments explore the unique oscillating contact line behaviors as affected by surface tension, gravity, inertial force and viscous force. The contact line exhibits both slip $(\sigma \rightarrow 0)$ and stick $(\sigma \rightarrow \infty)$ behavior over one oscillation period. To determine the dominant forces in each experiment, first we calculate the parameter $p=v \sqrt{k_{0}^{3} / g}$, measuring the primary viscous effect of the Stokes layer on the plate, as in the study of the waves produced by a vertically oscillating plate [7]. Viscosity is negligible provided that $p \ll 1$. The viscous effect parameter of our experiments is much less than one in each case, indicating that the inviscid approximation is valid. Second, we calculate another parameter $R_{\mathrm{sgi}}=\rho \hat{a}^{2}\left(g+\hat{a} \omega^{2}\right) / \sigma$, the ratio between the combination of gravity and

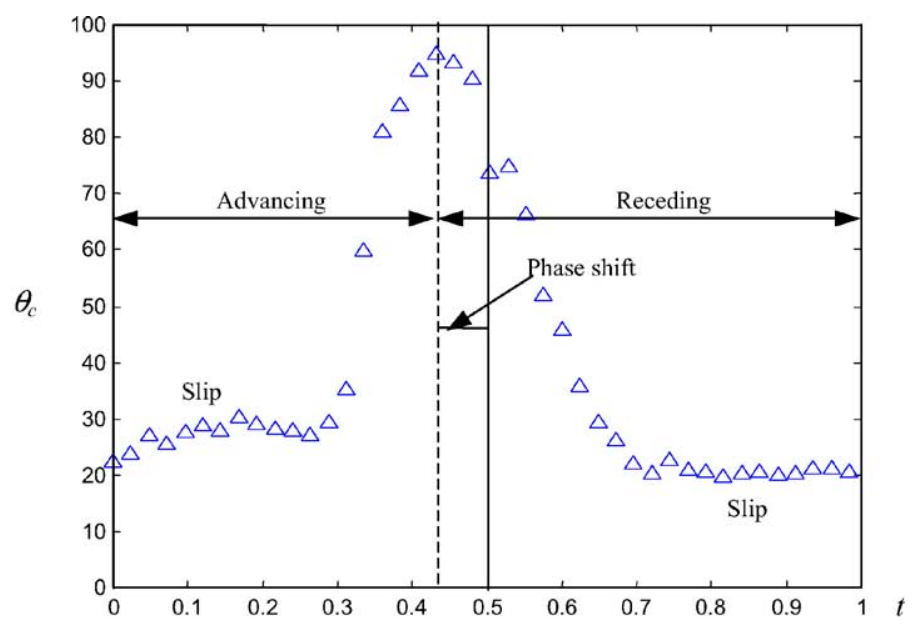

(a)

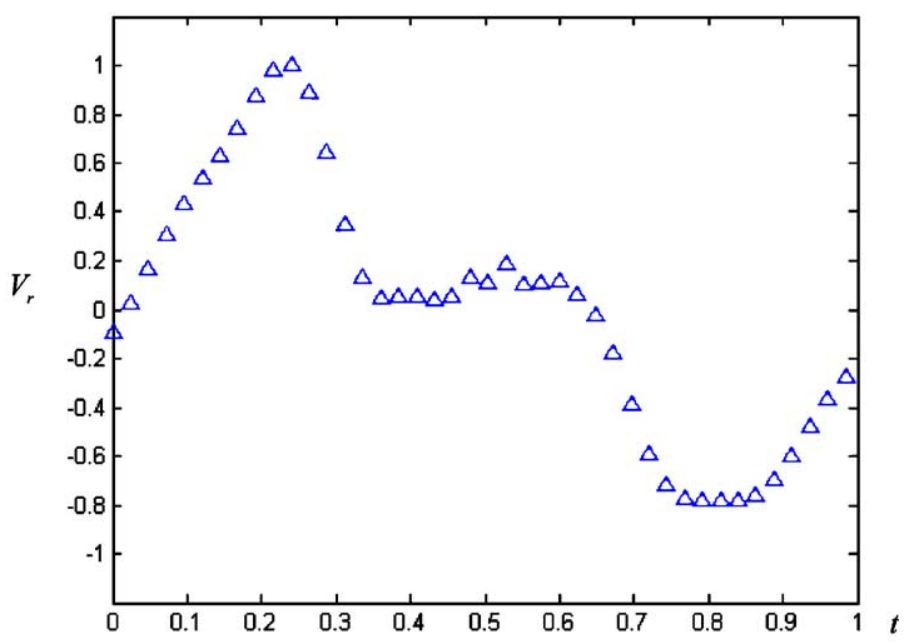

(b)

Fig. 11. Experimental evidence of the stick, partial stick and slip motions during one period. (a) Dynamic contact angle $\theta_{\mathrm{c}}$ and (b) relative contact line velocity $V_{\mathrm{r}}$ variation over one period. 
Table 3

$R_{\text {sgi }}$ number for each experiment

\begin{tabular}{|c|c|c|c|c|c|c|c|}
\hline \multirow[t]{2}{*}{ Frequency $(\mathrm{Hz})$} & \multicolumn{7}{|c|}{ Approximate stroke amplitude (mm) } \\
\hline & 0.5 & 1 & 2 & 3 & 4 & 5 & 6 \\
\hline 2 & 0.034 & 0.137 & 0.555 & 1.268 & 2.289 & 3.630 & 5.305 \\
\hline 4 & 0.035 & 0.143 & 0.607 & 1.443 & 2.704 & 4.441 & 6.707 \\
\hline 6 & 0.036 & 0.154 & 0.693 & 1.735 & 3.396 & 5.793 & 9.043 \\
\hline 8 & 0.038 & 0.169 & 0.814 & 2.144 & 4.365 & 7.686 & 12.31 \\
\hline 12 & 0.043 & 0.212 & 1.161 & 3.312 & 7.134 & - & - \\
\hline 16 & 0.051 & 0.273 & 1.645 & 4.948 & - & - & - \\
\hline
\end{tabular}

inertial forces to the surface tension. Both Ting and Perlin's and our experiments observe more obvious contact-line slip as the $R_{\text {sgi }}$ number increases as shown in Table 3, and is evident in Fig. 9 where $\hat{a}$ is increasing from the top graph to the bottom graph and hence so is $R_{\text {sgi }}$. (In graphs of contact angle versus relative velocity, data points not located on the $V_{\mathrm{r}}=0$ ordinate represent at least partial slip.)

Within one oscillation period, our experiments show that slip occurs mostly near the top of the plate stroke. This position is associated with the maximum acceleration in the same direction as gravity. Due to the opposite directions of the maximum acceleration and gravity, the possibility of slip at the bottom of the plate stroke is reduced. Fig. 11 shows that for oscillating flows a phase shift exists between the contact-line and plate motion. When slip occurs, the contact line moves freely along the plate and the contact angle barely changes. Between the highest and the lowest positions is the transition motion with the contact angle varying rapidly. Our experiments show that the advancing contact angle changes more rapidly than the receding contact angle.

Our experimental results of low Reynolds number oscillations are consistent with Benjamin and Scott's assumption that when the dynamic contact angle varies within the static range, the contact line is fixed. The pinned-edge condition can provide a good prediction.

For high Reynolds number oscillatory flows, a slip model with a time-dependent slip coefficient $c$ is required. The results show that the inviscid model with pinned-edge condition and the real static contact angle predicts the experimental data well; however, it fails to predict the contact line behavior adequately when slip occurs.

\section{References}

[1] C.-L. Ting, M. Perlin, Boundary conditions in the vicinity of the contact line at a vertically oscillating upright plate: an experimental investigation, J. Fluid Mech. 295 (1995) 263-300.

[2] T.B. Benjamin, J.C. Scott, Gravity-capillary waves with edge constraints, J. Fluid Mech. 92 (1979) 241-267.

[3] J. Graham-Eagle, Gravity-capillary waves with edge constraints, D.Phil. Thesis, University of Oxford, 1984.

[4] E.B. Dussan, On the spreading of liquids on solid surfaces: static and dynamic contact lines, Ann. Rev. Fluid Mech. 11 (1979) $371-400$.

[5] S.H. Davis, Contact-line problems in fluid mechanics, J. Appl. Mech. Trans ASME 50 (4b) (1983) 977-982.

[6] L.M. Hocking, The damping of capillary-gravity waves at a rigid boundary, J. Fluid Mech. 179 (1987) 253-266.

[7] L.M. Hocking, Waves produced by a vertically oscillating plate, J. Fluid Mech. 179 (1987) 267-281.

[8] G.W. Young, S.H. Davis, A plate oscillating across a liquid interface: effects of contact angle hysteresis, J. Fluid Mech. 174 (1987) $327-356$.

[9] J.W. Miles, Capillary-viscous forcing of surface waves, J. Fluid Mech. 219 (1990) 635-646.

[10] D.M. Henderson, J.W. Miles, Surface wave damping in a circular cylinder with a fixed contact line, J. Fluid Mech. 275 (1994) $285-299$.

[11] Z.Y. Liu, Film flow within an axially rotating horizontal cylinder and contact lines on an oscillating plate, Ph.D. Dissertation, University of Michigan, 2001.

[12] S.H. Davis, Moving contact lines and rivulet instabilities. I. The static rivulet, J. Fluid Mech. 98 (1980) $225-242$. 